\title{
Cell viability of acute myeloid leukaemia blasts in culture correlates with treatment outcome
}

\begin{abstract}
Despite the advances in understanding the pathophysiology of acute myeloid leukaemia (AML), the cure rate for acute myeloid leukaemia patients remains low. Cytogenetic abnormalities and age are the prognostic factors that guide treatment decisions. However, many AML patients still die. The biological factors that influence treatment outcome are largely unknown. Thus, the objective of our study was to use the in vitro viability test to correlate with treatment outcome. Acute myeloid leukaemia blasts demonstrated differing ability to survive in culture. Our examination of blast phenotype at various days in culture showed two possible growth directions. First, cells underwent maturation by increased expression of CD16 and down-regulated CD34 (a haemopoietic stem cell marker). These cells also appeared to have undergone apoptosis. Alternatively, cells continued to survive in culture and maintained high expression of CD34. An MTT assay was carried out to determine viability after three days of culture. Lower optical density values were obtained for samples that underwent apoptosis and higher values were obtained for samples that survived in culture. Apoptosis was measured by Annexin V/propidium iodide staining. A comparison between results of MTT assay and duration of disease free survival revealed that a higher viability in vitro correlated significantly with shorter survival duration in the patient $(\mathrm{R}=-$ $0.761, \mathrm{p}=0.002, \mathrm{n}=13$ ). Thus, this study further supports the hypothesis that AML patients with poor survival may be related to having blasts with a biologically more immature or stem cell-like nature.
\end{abstract}

Keyword: AML; Cell culture; MTT assay; Phenotype; Treatment outcome 